\title{
A Study to Analyze the Impact of Knowledge Management (KM) practices in IT Service Delivery Industry in India
}

\author{
Barakath Ali Shaik Mohamed $^{1}$, Ramanigopal C.S ${ }^{2}$ and P.M Murali
}

${ }^{1}$ Ph.D. Research Scholar, Research and Development Centre, Vinayaka Missions

University, Salem, Tamilnadu, India - 636308

2Professor and Head, Dean - Managemlent Studies, Faculty of Management Studies, Vinayaka Missions Kirupananda Variyar Engineering College, Salem, Tamilnadu, India - 636308

${ }^{3}$ Associate Professor - Management Studies, Faculty of Management Studies, Vinayaka Missions Kirupananda

Variyar Engineering College, Salem, Tamilnadu, India - 636308

Article History: Received:11 January 2021; Accepted: 27 February 2021; Published online: 5 April 2021

\begin{abstract}
Information Technology (IT) Industry in India is growing fast. Organizations across the world are outsourcing the IT services to take advantage of cost and time differences. India plays vital role in supporting the outsourcing services by taking advantage of the language skills and skilled workforce. Knowledge Management (KM) is critical for the continuity of the services and success of the organisations in different domains. This paper analysis the impact of Knowledge Management in Information Technology (IT) Service Delivery Industry in India. The study reveals Knowledge Management improves the performance of the Service Delivery organization. Methodical implementation and continuous improvement of Knowledge Management practices would further accelerate and improve the customer experience.
\end{abstract}

Key Words: Knowledge Management, Information Technology, Service Delivery Industry

\section{INTRODUCTION}

Global business environment becomes increasingly competitive day by day. There is a growing need for servicebased organizations to adopt the best practices, tools and methodologies as part of implementation of Information Technology Service Management (ITSM). Information Technology service provider organizations have either implemented or in the process of implementing the Information Technology Infrastructure Library (ITIL) framework to continuously measure and monitor their Information Technology operations to improve service delivery and customer satisfaction. Knowledge Administration is a critical step in Service evolution phase of Information Technology Service Management. While few larger service providers through experience have matured their service management process over the years, many small, medium, and few large organizations still face significant challenges in improving service management processes. Repeatable processes and services could be automated by combining various automation tools available in the market for knowledge management and Information Technology service management.

\subsection{Global Information Technology Industry}

Recent Market report of Gartner says that the spending of entire international IT sector that include IT solutions, data center systems, business applications and telecom providers is likely to exceed $\$ 3.7$ trillion during 2019, which shows an improvement of $4.5 \%$ over 2018. This growth in international IT sector started in 2018 and is expected to grow in the future too.

Today the greatest and fastest growing segment in the International IT sector is the Internet of Things (IoT). It has grown at an astonishing rate of $30 \%$ within a five-year span from $\$ 700$ million in 2016 and is expected to be over \$ 2 billion by 2021 .

\subsection{Information Technology Industry in India}

Information Technology in India is growing at a rapid pace. It comprises of two components namely - IT services and business process outsourcing (BPO). The sector has gone from contributing 1.2\% to India's GDP in 1998 to $7.7 \%$ in 2017 . Based on the data released by NASSCOM, the sector aggregated revenues of US\$180 billion in 2019 growing by over $13 \%$. The export revenue was recorded at US\$99 billion and domestic revenue at US\$48 billion. As of 2020, more than 4.36 million employees were accounted as India's IT workforce. United States is the largest employer accounting for about two-thirds of India's IT service exports.

\subsection{IT Service Delivery}

IT Service Delivery refers to the way in which an organisation provides users access to IT services that include Infrastructure, Applications, data storage and other business resources. They cover design, development, 
deployment, operation and retirement. These stages of service delivery are executed by IT professionals. Quality of IT service delivery is evaluated by metrics that are include in the service level agreement (SLA).

\subsection{Knowledge Management}

Knowledge management is the conscious process of defining, structuring, retaining and sharing the knowledge and experience of employees within an organization. The main goal of knowledge management is to improve an organization's efficiency and save knowledge within the company. Successful knowledge management will improve an organization in several ways. It will ensure that the specialized Knowledge of employees does not leave with them or go unutilized by other employees who would benefit from that knowledge. It allows for better situational awareness, as well as opening doors for learning about best practices, lessons learned, and overall organizational improvement.

\subsection{Knowledge Management in India}

Knowledge Management is a relatively new but a fast emerging concept in India. It promotes integrated approach to identifying, managing and sharing information assets of an organization. The information assets may include but are not restricted to databases, documents, policies, procedures and also expertise in employees.

\subsection{Knowledge Management in Organisations}

Organizations rely heavily on the Knowledge to support the business. Advances in technology, innovations and policies are practically every day evolving globally. Knowledge management (KM) is a serious and vibrant structural resource that helps in the global competitive business environment to reach competence, effectiveness and viable gain. In the new Digital Evolution Information Technology (IT) is a key driver of many business function. Knowledge Management is a key component of service management which plays the major role.

\section{OBJECTIVE}

A Study to analyze the impact of Knowledge Management (KM) practices in IT Service Delivery Industry in India. Large IT organizations with minimum of 50,000 employees and having offices in multiple locations providing 10+ years of service in India are considered for the study.

\section{METHODOLOGY}

We have used both primary and secondary data for this study. The primary data was gathered from stakeholders as part of weekly service delivery review meetings observations. Primary qualitative data was collected through observation due to time limitation and to minimize the cost of data collection. The secondary quantitative data was gathered from Service Now IT Service Management Configuration Management Data Base (CMDB). The category of Organizations taken for study are IT service delivery companies operating out of India. Most of the large IT service companies in India having more than 10 years with minimum of 50,000 employees and having offices in multiple locations in India are considered for the study. Research is being done as a part time basis, availability of researcher time, cost and efforts are limitations.

\section{ANALYSIS}

We have considered IT organizations having more than 50000 employees and operating out of multiple locations in India. Considering the time, efforts and cost, we have selected one IT organization for this study.We have taken 12 months primary and secondary ticket data for the analysis. The data categories contain both incident and Service request tickets. The KM implementation started prior to data analysis period and improvements have been continuously implemented on ongoing basis.

The below table-1 and graph-1 gives an overall monthly combined ticket trend for Incidents and Service requests serviced by the organization. The overall ticket volume is in decreasing trend due to KM implementation.

Table-1: Monthly ticket trend combined (Incidents and Service Requests)

\begin{tabular}{|c|c|c|c|c|c|c|c|c|c|c|c|c|c|}
\hline $\begin{array}{c}\text { Mont } \\
\text { h }\end{array}$ & $\begin{array}{c}\text { May- } \\
19\end{array}$ & $\begin{array}{c}\text { Jun- } \\
19\end{array}$ & $\begin{array}{c}\text { Jul- } \\
19\end{array}$ & $\begin{array}{c}\text { Aug- } \\
19\end{array}$ & $\begin{array}{c}\text { Sep- } \\
19\end{array}$ & $\begin{array}{c}\text { Oct- } \\
19\end{array}$ & $\begin{array}{c}\text { Nov- } \\
19\end{array}$ & $\begin{array}{c}\text { Dec- } \\
19\end{array}$ & $\begin{array}{c}\text { Jan- } \\
20\end{array}$ & $\begin{array}{c}\text { Feb- } \\
20\end{array}$ & $\begin{array}{c}\text { Mar- } \\
20\end{array}$ & $\begin{array}{c}\text { Apr- } \\
20\end{array}$ & $\begin{array}{c}\text { Gran } \\
\text { d } \\
\text { Total }\end{array}$ \\
\hline $\begin{array}{c}\text { Ticke } \\
\text { ts }\end{array}$ & 2045 & 2189 & 2098 & 1897 & 2058 & 1721 & 1865 & 1576 & 1456 & 1510 & 1397 & 1340 & $\begin{array}{c}2115 \\
2\end{array}$ \\
\hline
\end{tabular}


A Study to Analyze the Impact of Knowledge Management (KM) practices in IT Service Delivery Industry in India

Graph -1:Monthly ticket trend combined (Incidents and Service Requests)

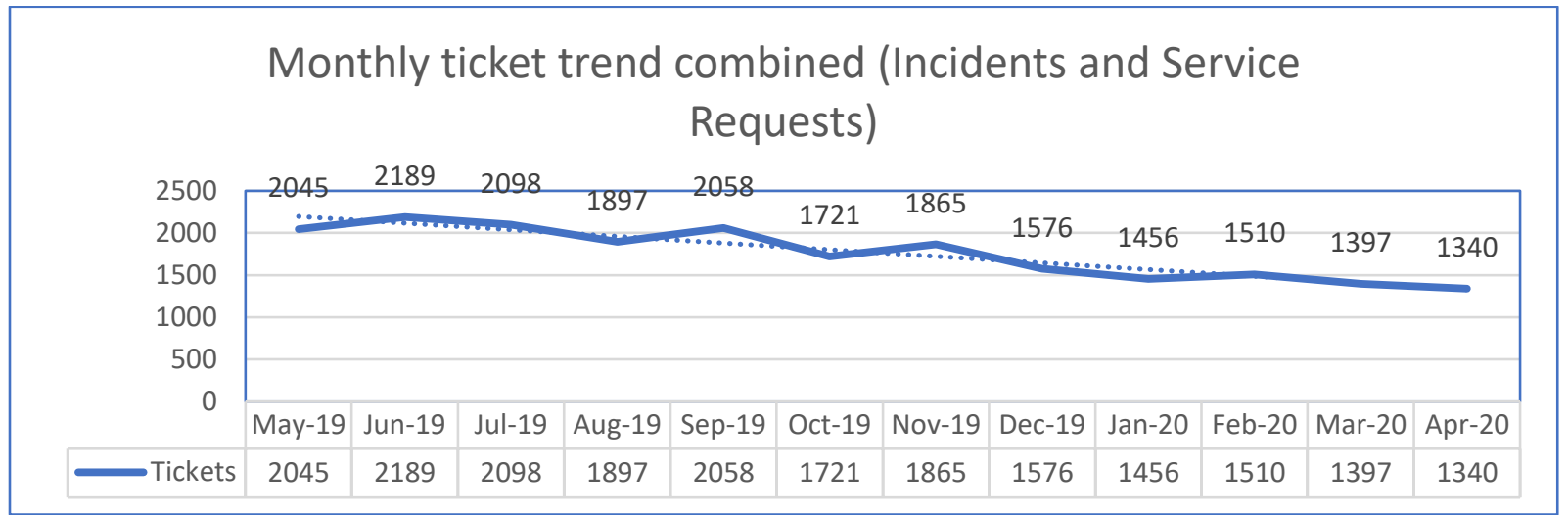

The below table-2 and graph-2 gives monthly ticket trend for Incidents and Service requests serviced by the organization. The overall ticket volume is in decreasing trend for both Incident and Service request. The incident is contributing to $64 \%$ of overall volume and service request is contributing to $36 \%$ of overall volume.

Table -2:Monthly ticket trend - Incidents and Service Requests

\begin{tabular}{|c|c|c|c|c|c|c|c|c|c|c|c|c|c|}
\hline Month & $\begin{array}{c}\text { May- } \\
19\end{array}$ & $\begin{array}{c}\text { Jun- } \\
19\end{array}$ & $\begin{array}{c}\text { Jul- } \\
19\end{array}$ & $\begin{array}{c}\text { Aug } \\
-19\end{array}$ & $\begin{array}{c}\text { Sep- } \\
19\end{array}$ & $\begin{array}{c}\text { Oct- } \\
19\end{array}$ & $\begin{array}{c}\text { Nov- } \\
19\end{array}$ & $\begin{array}{c}\text { Dec- } \\
19\end{array}$ & $\begin{array}{c}\text { Jan- } \\
20\end{array}$ & $\begin{array}{c}\text { Feb- } \\
20\end{array}$ & $\begin{array}{c}\text { Mar- } \\
20\end{array}$ & $\begin{array}{c}\text { Apr- } \\
20\end{array}$ & $\begin{array}{c}\text { Grand } \\
\text { Total }\end{array}$ \\
\hline Incident & 1443 & $\begin{array}{c}136 \\
6\end{array}$ & $\begin{array}{c}127 \\
1\end{array}$ & $\begin{array}{c}117 \\
9\end{array}$ & $\begin{array}{c}139 \\
9\end{array}$ & $\begin{array}{c}105 \\
9\end{array}$ & 1132 & $\begin{array}{c}106 \\
7\end{array}$ & 912 & 991 & 895 & 867 & 13581 \\
\hline $\begin{array}{c}\text { Service } \\
\text { Request }\end{array}$ & 602 & 823 & 827 & 718 & 659 & 662 & 733 & 509 & 544 & 519 & 502 & 473 & 7571 \\
\hline Grand Total & 2045 & $\begin{array}{c}218 \\
9\end{array}$ & $\begin{array}{c}209 \\
8\end{array}$ & $\begin{array}{c}189 \\
7\end{array}$ & $\begin{array}{c}205 \\
8\end{array}$ & $\begin{array}{c}172 \\
1\end{array}$ & 1865 & $\begin{array}{c}157 \\
6\end{array}$ & $\begin{array}{c}145 \\
6\end{array}$ & $\begin{array}{c}151 \\
0\end{array}$ & 1397 & $\begin{array}{c}134 \\
0\end{array}$ & 21152 \\
\hline
\end{tabular}

Graph-2: Monthly ticket trend - Incidents and Service Requests

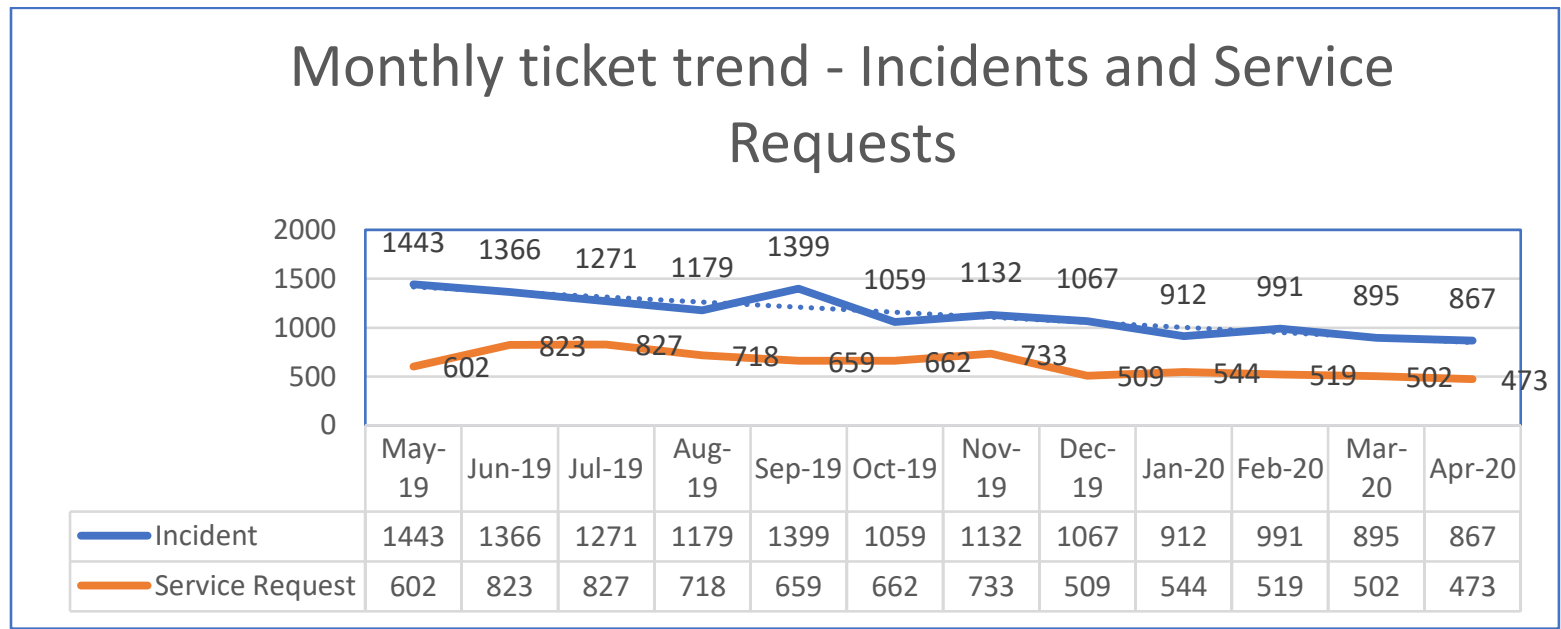

The below table- 3 and graph-3 gives an overall monthly combined ticket trend for Incidents and Service requests serviced by the organization by location. The volume contribution from Bangalore is $9 \%$, Chennai is $20 \%$, Hydrabad is $24 \%$, Indore is $9 \%$, Mumbai is $2 \%$ and Noida is $37 \%$. 
Table -3:Location wise Monthly ticket trend combined (Incidents and Service Requests)

\begin{tabular}{|l|r|r|r|r|r|r|r|r|r|r|r|r|r|}
\hline Month & $\begin{array}{l}\text { May- } \\
19\end{array}$ & $\begin{array}{l}\text { Jun- } \\
19\end{array}$ & $\begin{array}{l}\text { Jul- } \\
19\end{array}$ & $\begin{array}{l}\text { Aug- } \\
19\end{array}$ & $\begin{array}{l}\text { Sep- } \\
19\end{array}$ & $\begin{array}{l}\text { Oct- } \\
19\end{array}$ & $\begin{array}{l}\text { Nov- } \\
19\end{array}$ & $\begin{array}{l}\text { Dec- } \\
19\end{array}$ & $\begin{array}{l}\text { Jan- } \\
20\end{array}$ & $\begin{array}{l}\text { Feb- } \\
20\end{array}$ & $\begin{array}{l}\text { Mar- } \\
20\end{array}$ & $\begin{array}{l}\text { Apr } \\
-20\end{array}$ & $\begin{array}{l}\text { Grand } \\
\text { Total }\end{array}$ \\
\hline $\begin{array}{l}\text { Bangalor } \\
\text { e }\end{array}$ & 129 & 168 & 224 & 165 & 215 & 180 & 154 & 135 & 117 & 128 & 119 & 107 & 1841 \\
\hline Chennai & 411 & 431 & 535 & 403 & 383 & 353 & 368 & 303 & 266 & 289 & 287 & 274 & 4303 \\
\hline $\begin{array}{l}\text { Hyderaba } \\
\text { d }\end{array}$ & 524 & 555 & 441 & 405 & 488 & 421 & 454 & 385 & 352 & 346 & 340 & 326 & 5037 \\
\hline Indore & 198 & 212 & 189 & 149 & 176 & 146 & 156 & 126 & 113 & 119 & 123 & 117 & 1824 \\
\hline Mumbai & 34 & 42 & 29 & 31 & 41 & 25 & 34 & 34 & 26 & 31 & 27 & 23 & 377 \\
\hline Noida & 749 & 781 & 680 & 744 & 755 & 596 & 699 & 593 & 582 & 597 & 501 & 493 & 7770 \\
\hline $\begin{array}{l}\text { Grand } \\
\text { Total }\end{array}$ & 2045 & 2189 & 2098 & 1897 & $\begin{array}{r}205 \\
8\end{array}$ & $\begin{array}{r}172 \\
1\end{array}$ & 1865 & $\begin{array}{r}157 \\
6\end{array}$ & $\begin{array}{r}145 \\
6\end{array}$ & $\begin{array}{r}151 \\
0\end{array}$ & 1397 & $\begin{array}{r}134 \\
0\end{array}$ & 21152 \\
\hline
\end{tabular}

Graph-3: Location wise Monthly ticket trend combined (Incidents and Service Requests)

\section{Location wise Monthly ticket trend combined (Incidents and Service Requests)}

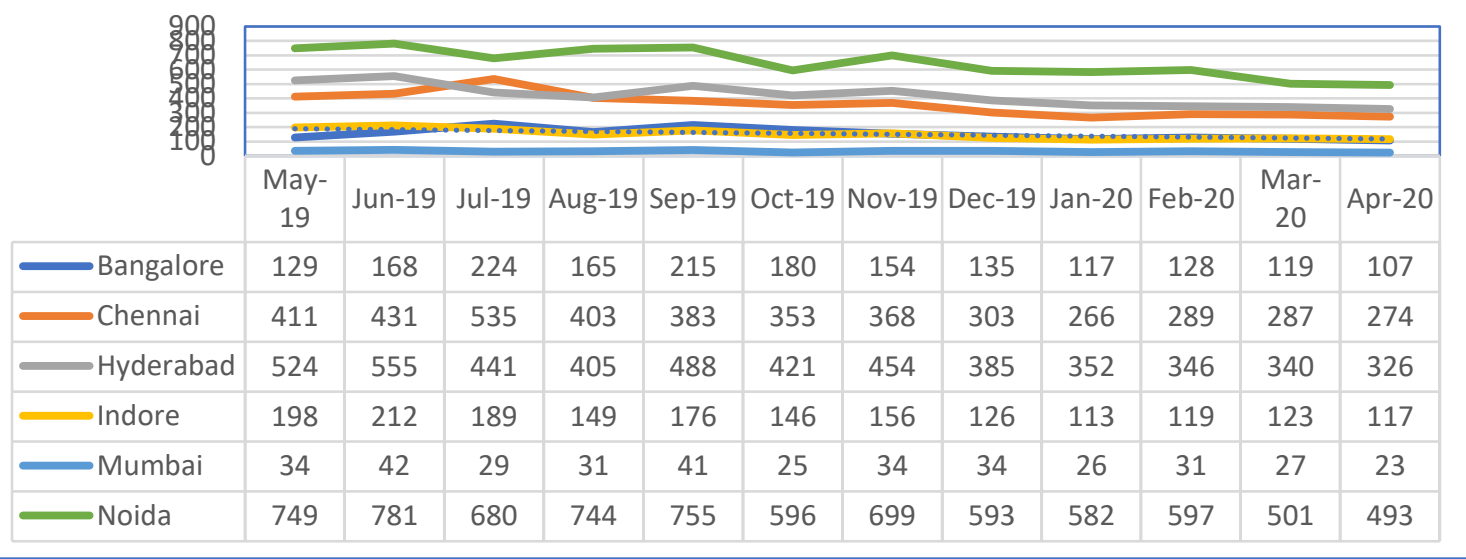

The below table- 4 and graph-4 gives monthly ticket trend for Incidents serviced by the organization by location. The volume contribution from Bangalore is $7 \%$, Chennai is $21 \%$, Hydrabad is $25 \%$, Indore is $9 \%$, Mumbai is $2 \%$ and Noida is $36 \%$.

Table -4: Location wise Monthly ticket trend - Incidents

\begin{tabular}{|l|r|l|l|r|r|r|r|r|r|r|r|r|r|}
\hline Month & $\begin{array}{l}\text { May- } \\
19\end{array}$ & $\begin{array}{l}\text { Jun- } \\
19\end{array}$ & $\begin{array}{l}\text { Jul- } \\
19\end{array}$ & $\begin{array}{l}\text { Aug- } \\
19\end{array}$ & $\begin{array}{l}\text { Sep- } \\
19\end{array}$ & $\begin{array}{l}\text { Oct- } \\
19\end{array}$ & $\begin{array}{l}\text { Nov- } \\
19\end{array}$ & $\begin{array}{l}\text { Dec- } \\
19\end{array}$ & $\begin{array}{l}\text { Jan- } \\
20\end{array}$ & $\begin{array}{l}\text { Feb- } \\
20\end{array}$ & $\begin{array}{l}\text { Mar- } \\
20\end{array}$ & $\begin{array}{l}\text { Apr } \\
-20\end{array}$ & $\begin{array}{l}\text { Grand } \\
\text { Total }\end{array}$ \\
\hline Incident & 1443 & 1366 & 1271 & 1179 & $\begin{array}{r}139 \\
9\end{array}$ & $\begin{array}{r}105 \\
9\end{array}$ & 1132 & 1067 & 912 & 991 & 895 & 867 & 13581 \\
\hline $\begin{array}{l}\text { Bangalo } \\
\text { re }\end{array}$ & 72 & 99 & 96 & 95 & 98 & 93 & 91 & 70 & 68 & 73 & 62 & 64 & 981 \\
\hline Chennai & 289 & 267 & 260 & 224 & 284 & 182 & 224 & 256 & 214 & 249 & 214 & 203 & 2866 \\
\hline $\begin{array}{l}\text { Hyderab } \\
\text { ad }\end{array}$ & 393 & 341 & 256 & 253 & 337 & 285 & 316 & 262 & 248 & 253 & 215 & 210 & 3369 \\
\hline Indore & 136 & 129 & 146 & 101 & 149 & 106 & 136 & 77 & 68 & 66 & 63 & 62 & 1239 \\
\hline Mumbai & 23 & 34 & 24 & 21 & 38 & 16 & 27 & 30 & 21 & 27 & 16 & 19 & 296 \\
\hline Noida & 530 & 496 & 489 & 485 & 493 & 377 & 338 & 372 & 293 & 323 & 325 & 309 & 4830 \\
\hline
\end{tabular}


A Study to Analyze the Impact of Knowledge Management (KM) practices in IT Service Delivery Industry in India

Graph-4: Location wise Monthly ticket trend - Incidents

\begin{tabular}{|c|c|c|c|c|c|c|c|c|c|c|c|c|}
\hline \multirow{2}{*}{ 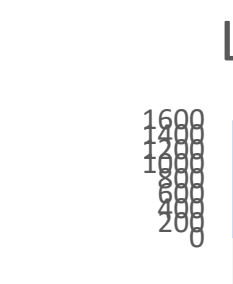 } & \multicolumn{12}{|c|}{ Location wise Monthly ticket trend - Incidents } \\
\hline & May-19 & Jun-19 & Jul-19 & Aug-19 & Sep-19 & Oct-19 & Nov-19 & Dec-19 & Jan-20 & Feb-20 & Mar-20 & Apr-20 \\
\hline Incident & 1443 & 1366 & 1271 & 1179 & 1399 & 1059 & 1132 & 1067 & 912 & 991 & 895 & 867 \\
\hline Bangalore & 72 & 99 & 96 & 95 & 98 & 93 & 91 & 70 & 68 & 73 & 62 & 64 \\
\hline Chennai & 289 & 267 & 260 & 224 & 284 & 182 & 224 & 256 & 214 & 249 & 214 & 203 \\
\hline Hyderabad & 393 & 341 & 256 & 253 & 337 & 285 & 316 & 262 & 248 & 253 & 215 & 210 \\
\hline Indore & 136 & 129 & 146 & 101 & 149 & 106 & 136 & 77 & 68 & 66 & 63 & 62 \\
\hline Mumbai & 23 & 34 & 24 & 21 & 38 & 16 & 27 & 30 & 21 & 27 & 16 & 19 \\
\hline$\longrightarrow$ Noida & 530 & 496 & 489 & 485 & 493 & 377 & 338 & 372 & 293 & 323 & 325 & 309 \\
\hline
\end{tabular}

The below table-5 and graph-5 gives monthly ticket trend for Service request serviced by the organization by location. The volume contribution from Bangalore is $11 \%$, Chennai is $19 \%$, Hydrabad is $22 \%$, Indore is $8 \%$, Mumbai is $1 \%$ and Noida is $39 \%$.

Table -5: Location wise Monthly ticket trend - Service Requests

\begin{tabular}{|l|r|l|l|l|l|r|r|l|l|r|r|r|r|}
\hline Month & $\begin{array}{l}\text { May- } \\
19\end{array}$ & $\begin{array}{l}\text { Jun- } \\
19\end{array}$ & $\begin{array}{l}\text { Jul- } \\
19\end{array}$ & $\begin{array}{l}\text { Aug } \\
-19\end{array}$ & $\begin{array}{l}\text { Sep- } \\
19\end{array}$ & $\begin{array}{l}\text { Oct- } \\
19\end{array}$ & $\begin{array}{l}\text { Nov- } \\
19\end{array}$ & $\begin{array}{l}\text { Dec- } \\
19\end{array}$ & $\begin{array}{l}\text { Jan- } \\
20\end{array}$ & $\begin{array}{l}\text { Feb- } \\
20\end{array}$ & $\begin{array}{l}\text { Mar- } \\
20\end{array}$ & $\begin{array}{l}\text { Apr } \\
-20\end{array}$ & Grand Total \\
\hline $\begin{array}{l}\text { Service } \\
\text { Request }\end{array}$ & 602 & 823 & 827 & 718 & 659 & 662 & 733 & 509 & 544 & 519 & 502 & 473 & 7571 \\
\hline Bangalore & 57 & 69 & 128 & 70 & 117 & 87 & 63 & 65 & 49 & 55 & 57 & 43 & 860 \\
\hline Chennai & 122 & 164 & 275 & 179 & 99 & 171 & 144 & 47 & 52 & 40 & 73 & 71 & 1437 \\
\hline $\begin{array}{l}\text { Hyderaba } \\
\text { d }\end{array}$ & 131 & 214 & 185 & 152 & 151 & 136 & 138 & 123 & 104 & 93 & 125 & 116 & 1668 \\
\hline Indore & 62 & 83 & 43 & 48 & 27 & 40 & 20 & 49 & 45 & 53 & 60 & 55 & 585 \\
\hline Mumbai & 11 & 8 & 5 & 10 & 3 & 9 & 7 & 4 & 5 & 4 & 11 & 4 & 81 \\
\hline Noida & 219 & 285 & 191 & 259 & 262 & 219 & 361 & 221 & 289 & 274 & 176 & 184 & 2940 \\
\hline
\end{tabular}

Graph-5: Location wise Monthly ticket trend - Service Requests

\section{Location wise Monthly ticket trend - Service Requests}

\begin{tabular}{|c|c|c|c|c|c|c|c|c|c|c|c|c|}
\hline & Mav-19 & lun-19 & -19 & Aug-19 & Sen-10 & Oct-19 & Nov-19 & & & & & \\
\hline - Service Request & 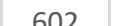 & 823 & 827 & 718 & 659 & 662 & 733 & 500 & 540 & 510 & 507 & 172 \\
\hline Bangalore & 57 & 69 & 128 & 70 & 117 & 87 & 63 & 65 & 49 & 55 & 57 & 43 \\
\hline Chennai & 122 & 164 & 275 & 179 & 99 & 171 & 144 & 47 & 52 & 40 & 73 & 71 \\
\hline Hyderabad & 131 & 214 & 185 & 152 & 151 & 136 & 138 & 123 & 104 & 93 & 125 & 116 \\
\hline Indore & 62 & 83 & 43 & 48 & 27 & 40 & 20 & 49 & 45 & 53 & 60 & 55 \\
\hline Mumbai & 11 & 8 & 5 & 10 & 3 & 9 & 7 & 4 & 5 & 4 & 11 & 4 \\
\hline Noida & 219 & 285 & 191 & 259 & 262 & 219 & 361 & 221 & 289 & 274 & 176 & 184 \\
\hline
\end{tabular}


The below table- 6 and graph- 6 gives monthly ticket trend for Incidents -High level categories serviced by the organization. The volume contribution for Application software is 2\%, Data center is 15\%, End user computing is $32.5 \%$, Interface Infra is 3\%, Network is $14 \%$, Security Software is $0.5 \%$ and System Software is $33 \%$.

Table -6: Monthly ticket trend - Incidents (High level Categories)

\begin{tabular}{|l|r|r|r|r|r|r|r|r|r|r|r|r|r|}
\hline Month & $\begin{array}{l}\text { May- } \\
19\end{array}$ & $\begin{array}{l}\text { Jun- } \\
19\end{array}$ & $\begin{array}{l}\text { Jul- } \\
19\end{array}$ & $\begin{array}{l}\text { Aug } \\
-19\end{array}$ & $\begin{array}{l}\text { Sep- } \\
19\end{array}$ & $\begin{array}{l}\text { Oct- } \\
19\end{array}$ & $\begin{array}{l}\text { Nov- } \\
19\end{array}$ & $\begin{array}{l}\text { Dec- } \\
19\end{array}$ & $\begin{array}{l}\text { Jan- } \\
20\end{array}$ & $\begin{array}{l}\text { Feb- } \\
20\end{array}$ & $\begin{array}{l}\text { Mar- } \\
20\end{array}$ & $\begin{array}{l}\text { Apr } \\
-20\end{array}$ & $\begin{array}{l}\text { Gran } \\
\text { d } \\
\text { Total }\end{array}$ \\
\hline $\begin{array}{l}\text { Incident } \\
1443\end{array}$ & $\begin{array}{r}136 \\
6\end{array}$ & $\begin{array}{r}127 \\
1\end{array}$ & $\begin{array}{r}117 \\
9\end{array}$ & $\begin{array}{r}139 \\
9\end{array}$ & $\begin{array}{r}105 \\
9\end{array}$ & 1132 & 1067 & 912 & 991 & 895 & 867 & $\begin{array}{r}1145 \\
0\end{array}$ \\
\hline $\begin{array}{l}\text { Application } \\
\text { Software }\end{array}$ & 35 & 32 & 17 & 31 & 18 & 21 & 17 & 14 & 11 & 3 & 3 & 192 \\
\hline Data center & 259 & 229 & 201 & 158 & 219 & 158 & 162 & 165 & 124 & 149 & 161 & 156 & 1710 \\
\hline $\begin{array}{l}\text { End user } \\
\text { computing }\end{array}$ & 543 & 498 & 415 & 379 & 499 & 363 & 384 & 359 & 311 & 333 & 270 & 264 & 3735 \\
\hline Interface Infra & 48 & 41 & 34 & 74 & 45 & 17 & 13 & 20 & 21 & 24 & 29 & 26 & 314 \\
\hline Network & 311 & 256 & 223 & 198 & 227 & 165 & 196 & 156 & 138 & 133 & 102 & 97 & 1631 \\
\hline $\begin{array}{l}\text { Security } \\
\text { Software }\end{array}$ & 5 & 5 & 3 & 5 & 7 & 3 & 4 & 2 & 1 & 1 & 3 & 2 & 42 \\
\hline $\begin{array}{l}\text { System } \\
\text { Software }\end{array}$ & 242 & 304 & 363 & 348 & 371 & 335 & 352 & 348 & 303 & 340 & 327 & 319 & 3826 \\
\hline
\end{tabular}

Graph-6: Monthly ticket trend - Incidents (High level Categories)

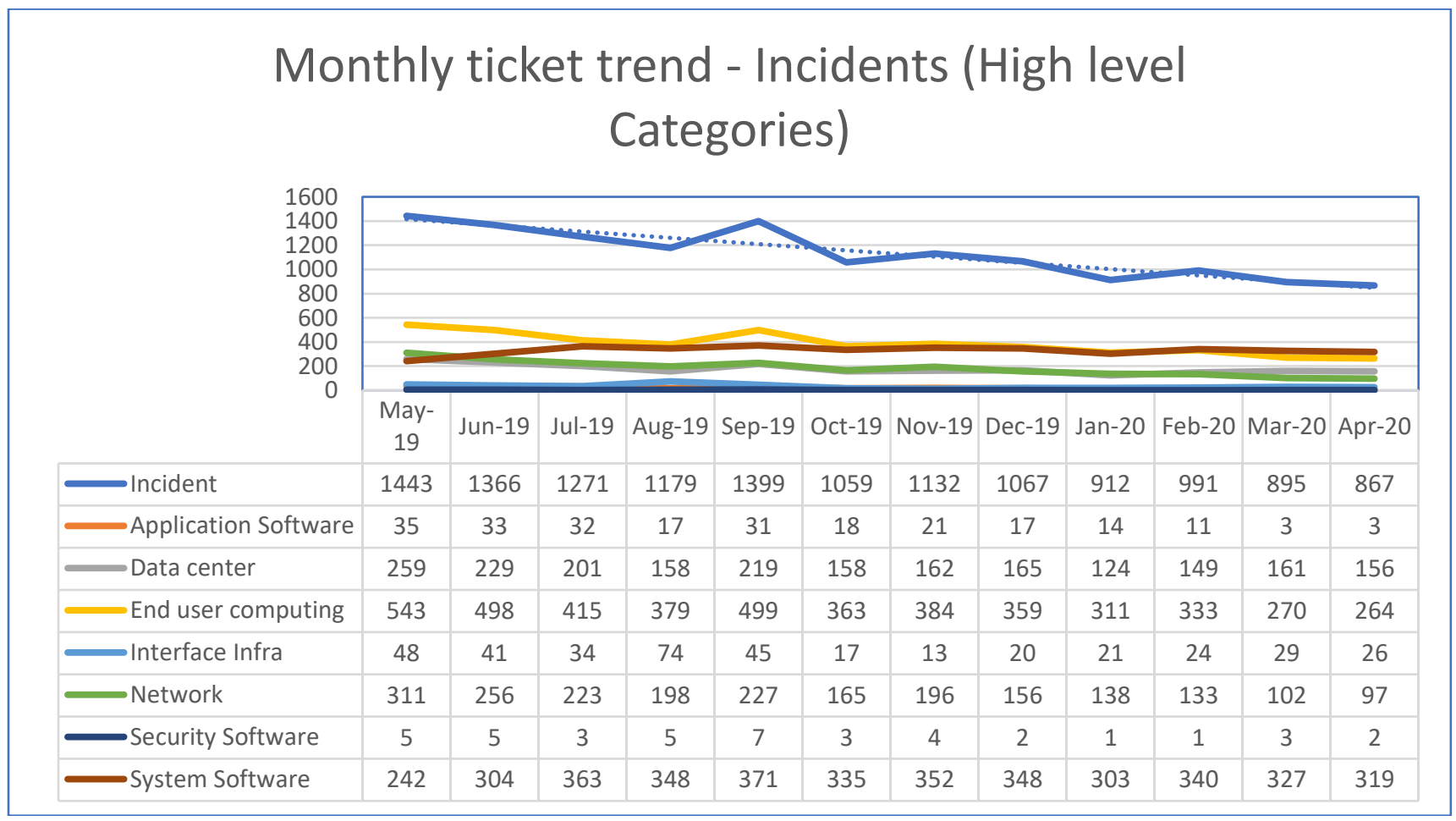

The below table-7 and graph-7 gives monthly ticket trend for Service request - High level categories serviced by the organization. The volume contribution for Application software is $47 \%$, End user computing is $45 \%$, Middleware software is $1 . \%$,Network-Vpn Client is $2 \%$ and Hardware upgradation is $5 \%$. 
A Study to Analyze the Impact of Knowledge Management (KM) practices in IT Service Delivery Industry in India

Table -7: Monthly ticket trend - Service Requests (High level Categories)

\begin{tabular}{|l|r|r|r|r|r|r|r|r|r|r|r|r|r|}
\hline Month & $\begin{array}{l}\text { May- } \\
19\end{array}$ & $\begin{array}{l}\text { Jun- } \\
19\end{array}$ & $\begin{array}{l}\text { Jul- } \\
19\end{array}$ & $\begin{array}{l}\text { Aug } \\
-19\end{array}$ & $\begin{array}{l}\text { Sep- } \\
19\end{array}$ & $\begin{array}{l}\text { Oct- } \\
19\end{array}$ & $\begin{array}{l}\text { Nov- } \\
19\end{array}$ & $\begin{array}{l}\text { Dec- } \\
19\end{array}$ & $\begin{array}{l}\text { Jan- } \\
20\end{array}$ & $\begin{array}{l}\text { Feb- } \\
20\end{array}$ & $\begin{array}{l}\text { Mar- } \\
20\end{array}$ & $\begin{array}{l}\text { Apr } \\
-20\end{array}$ & $\begin{array}{l}\text { Grand } \\
\text { Total }\end{array}$ \\
\hline Service Request & 602 & 823 & 827 & 718 & 659 & 662 & 733 & 509 & 544 & 519 & 502 & 473 & 7571 \\
\hline $\begin{array}{l}\text { Application } \\
\text { Software }\end{array}$ & 269 & 368 & 360 & 336 & 310 & 316 & 360 & 255 & 282 & 252 & 238 & 222 & 3568 \\
\hline $\begin{array}{l}\text { End user } \\
\text { computing }\end{array}$ & 270 & 392 & 393 & 332 & 310 & 280 & 307 & 228 & 236 & 231 & 218 & 209 & 3406 \\
\hline $\begin{array}{l}\text { Middleware } \\
\text { software }\end{array}$ & 5 & 9 & 7 & 3 & 1 & 5 & 4 & 2 & 2 & 3 & 1 & 1 & 43 \\
\hline $\begin{array}{l}\text { Network-VPN } \\
\text { Client }\end{array}$ & 14 & 18 & 14 & 3 & 9 & 17 & 13 & 3 & 1 & 7 & 18 & 16 & 133 \\
\hline $\begin{array}{l}\text { Hardware } \\
\text { upgradation }\end{array}$ & 44 & 36 & 53 & 44 & 29 & 44 & 49 & 21 & 23 & 26 & 27 & 25 & 421 \\
\hline
\end{tabular}

Graph-7: Monthly ticket trend - Service Requests (High level Categories)

\section{Monthly ticket trend - Service Requests (High level Categories)}

\begin{tabular}{|c|c|c|c|c|c|c|c|c|c|c|c|c|}
\hline 8 影目 & 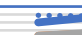 & $=$ & nominar & $=$ & & & inmengen & En & & & & 8 \\
\hline & $\begin{array}{c}\text { May- } \\
19\end{array}$ & $\begin{array}{c}\text { Jun- } \\
19\end{array}$ & Jul-19 & $\begin{array}{c}\text { Aug- } \\
19\end{array}$ & $\begin{array}{c}\text { Sep- } \\
19\end{array}$ & $\begin{array}{c}\text { Oct- } \\
19\end{array}$ & $\begin{array}{c}\text { Nov- } \\
19\end{array}$ & $\begin{array}{c}\text { Dec- } \\
19\end{array}$ & Jan-20 & $\begin{array}{c}\text { Feb- } \\
20\end{array}$ & $\begin{array}{c}\text { Mar- } \\
20\end{array}$ & $\begin{array}{c}\text { Apr- } \\
20\end{array}$ \\
\hline Service Request & 602 & 823 & 827 & 718 & 659 & 662 & 733 & 509 & 544 & 519 & 502 & 473 \\
\hline Application Software & 269 & 368 & 360 & 336 & 310 & 316 & 360 & 255 & 282 & 252 & 238 & 222 \\
\hline End user computing & 270 & 392 & 393 & 332 & 310 & 280 & 307 & 228 & 236 & 231 & 218 & 209 \\
\hline Middleware software & 5 & 9 & 7 & 3 & 1 & 5 & 4 & 2 & 2 & 3 & 1 & 1 \\
\hline Network-VPN Client & 14 & 18 & 14 & 3 & 9 & 17 & 13 & 3 & 1 & 7 & 18 & 16 \\
\hline Hardware upgradation & 44 & 36 & 53 & 44 & 29 & 44 & 49 & 21 & 23 & 26 & 27 & 25 \\
\hline
\end{tabular}

The below table- 8 and graph- 8 gives an overall monthly Knowledge articles created, and knowledge article utilized by the support team. The articles created and utilized percentage is in increasing trend as part of $\mathrm{KM}$ implementation.

Table -8: Monthly Knowledge Article Creation \& Usage trend

\begin{tabular}{|l|r|r|r|r|r|r|r|r|r|r|r|r|r|}
\hline Month & $\begin{array}{l}\text { May- } \\
19\end{array}$ & $\begin{array}{l}\text { Jun- } \\
19\end{array}$ & $\begin{array}{l}\text { Jul- } \\
19\end{array}$ & $\begin{array}{l}\text { Aug } \\
-19\end{array}$ & $\begin{array}{l}\text { Sep- } \\
19\end{array}$ & $\begin{array}{l}\text { Oct- } \\
19\end{array}$ & $\begin{array}{l}\text { Nov- } \\
19\end{array}$ & $\begin{array}{l}\text { Dec- } \\
19\end{array}$ & $\begin{array}{l}\text { Jan- } \\
20\end{array}$ & $\begin{array}{l}\text { Feb- } \\
20\end{array}$ & $\begin{array}{l}\text { Mar- } \\
20\end{array}$ & $\begin{array}{l}\text { Apr } \\
-20\end{array}$ & $\begin{array}{l}\text { Grand } \\
\text { Total }\end{array}$ \\
\hline $\begin{array}{l}\text { KA } \\
\text { Created }\end{array}$ & 50 & 60 & 78 & 96 & 125 & 165 & 204 & 273 & 338 & 423 & 547 & 610 & 2969 \\
\hline $\begin{array}{l}\text { KA } \\
\text { Utilized }\end{array}$ & 2 & 5 & 8 & 14 & 20 & 32 & 45 & 54 & 67 & 89 & 95 & 112 & 543 \\
\hline
\end{tabular}




\section{Graph-8: Monthly Knowledge Article Creation \& Usage trend}

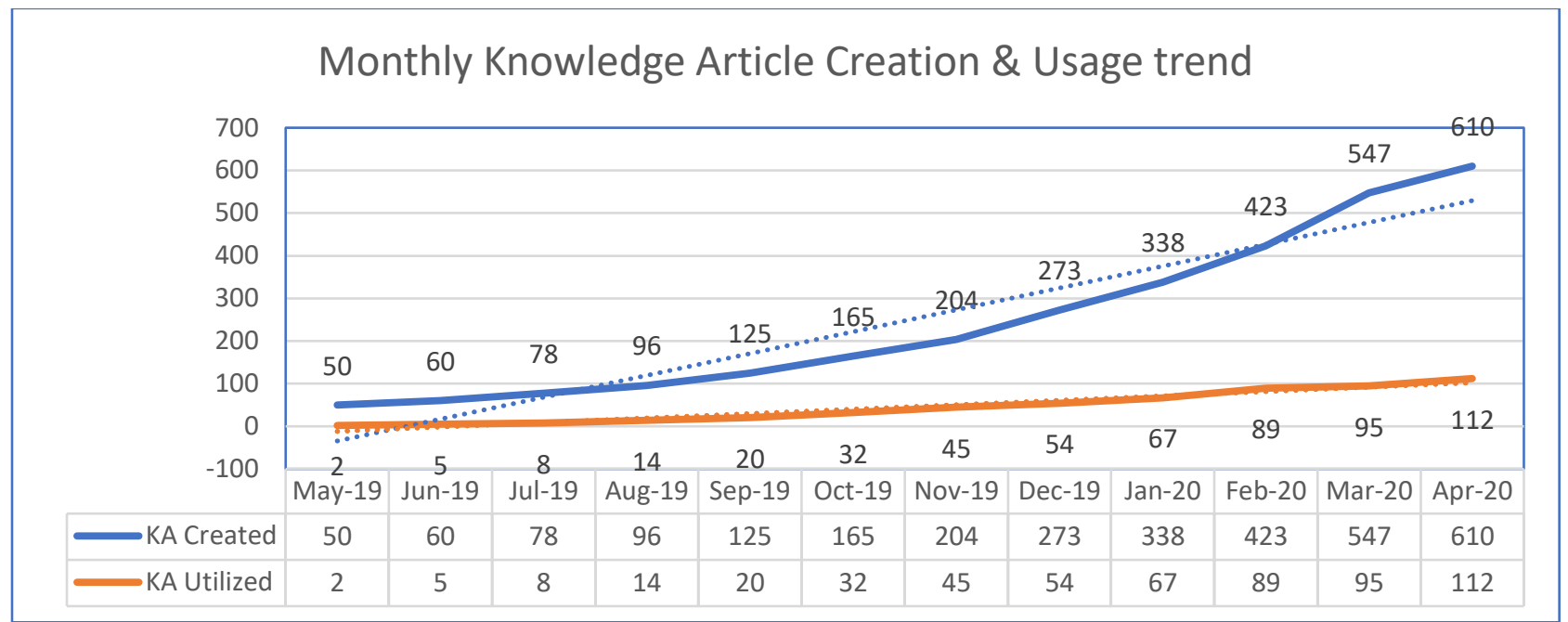

The below table-9 and graph-9 gives an overall monthly customer satisfaction trend for an year. The customer satisfaction level is increasing on month on month basis.

Table -9: Monthly Customer Satisfaction trend

\begin{tabular}{|l|l|l|l|l|l|l|l|l|r|r|r|r|l|}
\hline $\begin{array}{l}\text { Mont } \\
\text { h }\end{array}$ & $\begin{array}{l}\text { May- } \\
19\end{array}$ & $\begin{array}{l}\text { Jun- } \\
19\end{array}$ & $\begin{array}{l}\text { Jul- } \\
19\end{array}$ & $\begin{array}{l}\text { Aug- } \\
19\end{array}$ & $\begin{array}{l}\text { Sep- } \\
19\end{array}$ & $\begin{array}{l}\text { Oct- } \\
19\end{array}$ & $\begin{array}{l}\text { Nov- } \\
19\end{array}$ & $\begin{array}{l}\text { Dec- } \\
19\end{array}$ & $\begin{array}{l}\text { Jan- } \\
20\end{array}$ & $\begin{array}{l}\text { Feb- } \\
20\end{array}$ & $\begin{array}{l}\text { Mar- } \\
20\end{array}$ & $\begin{array}{l}\text { Apr } \\
-20\end{array}$ & $\begin{array}{l}\text { Grand } \\
\text { Total }\end{array}$ \\
\hline $\begin{array}{l}\text { CSA } \\
\text { T }\end{array}$ & 75 & 72 & 68 & 77 & 81 & 75 & 86 & 88 & 79 & 91 & 90 & 92 & 974 \\
\hline
\end{tabular}

\section{Graph-9: Monthly Customer Satisfaction trend}

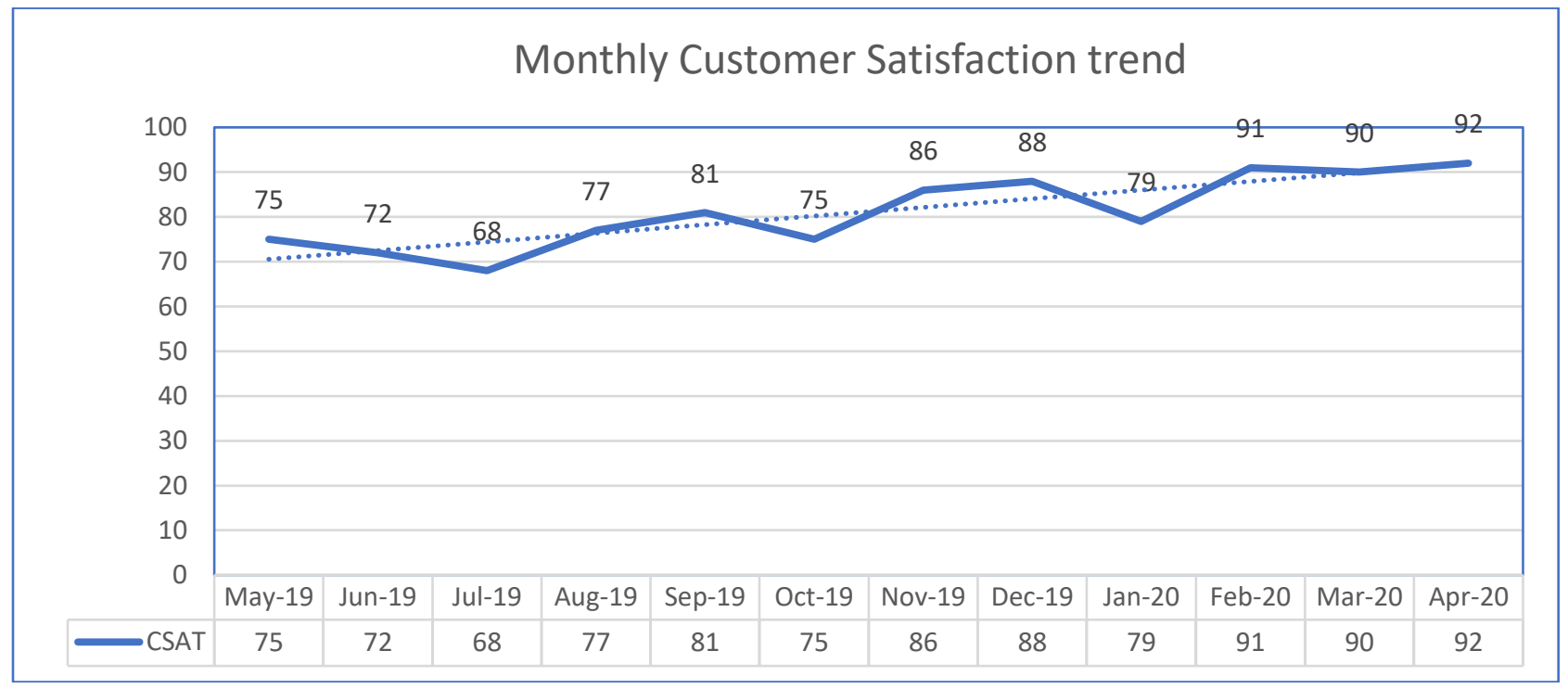

The below table-10 and graph-10 gives an overall monthly customer contact channel ticket trend for Incidents and Service requests serviced by the organization. The volume contribution for Voice is 7\%, Chat is $74 \%$, Email is $15 \%$ and Self Service is $4 \%$. 
A Study to Analyze the Impact of Knowledge Management (KM) practices in IT Service Delivery Industry in India

Table -10: Monthly Contact Channel trend combined (Incidents \& Service Requests)

\begin{tabular}{|c|c|c|c|c|c|c|c|c|c|c|c|c|c|}
\hline Month & $\begin{array}{l}\text { May- } \\
19\end{array}$ & $\begin{array}{l}\text { Jun- } \\
19\end{array}$ & $\begin{array}{l}\text { Jul- } \\
19\end{array}$ & $\begin{array}{l}\text { Aug } \\
-19\end{array}$ & $\begin{array}{l}\text { Sep- } \\
19\end{array}$ & $\begin{array}{l}\text { Oct- } \\
19\end{array}$ & $\begin{array}{l}\text { Nov- } \\
19\end{array}$ & $\begin{array}{l}\text { Dec- } \\
19\end{array}$ & $\begin{array}{l}\text { Jan- } \\
20\end{array}$ & $\begin{array}{l}\text { Feb- } \\
20\end{array}$ & $\begin{array}{l}\text { Mar- } \\
20\end{array}$ & $\begin{array}{l}\text { Apr } \\
-20\end{array}$ & $\begin{array}{l}\text { Grand } \\
\text { Total }\end{array}$ \\
\hline Tickets & 2045 & $\begin{array}{r}218 \\
9\end{array}$ & $\begin{array}{r}209 \\
8\end{array}$ & $\begin{array}{r}189 \\
7\end{array}$ & $\begin{array}{r}205 \\
8\end{array}$ & $\begin{array}{r}172 \\
1\end{array}$ & 1865 & 1576 & $\begin{array}{r}145 \\
6\end{array}$ & $\begin{array}{r}151 \\
0\end{array}$ & 1397 & $\begin{array}{r}134 \\
0\end{array}$ & 21152 \\
\hline Voice & 198 & 114 & 142 & 114 & 156 & 130 & 97 & 57 & 77 & 117 & 122 & 89 & 1413 \\
\hline Chat & 1525 & $\begin{array}{r}174 \\
6\end{array}$ & $\begin{array}{r}164 \\
4\end{array}$ & $\begin{array}{r}147 \\
6 \\
\end{array}$ & $\begin{array}{r}154 \\
9\end{array}$ & $\begin{array}{r}129 \\
4\end{array}$ & 1365 & 1140 & $\begin{array}{r}104 \\
8\end{array}$ & $\begin{array}{r}103 \\
7\end{array}$ & 916 & 879 & 15619 \\
\hline Email & 290 & 294 & 274 & 263 & 303 & 235 & 328 & 295 & 234 & 237 & 234 & 230 & 3217 \\
\hline $\begin{array}{l}\text { Self } \\
\text { Service }\end{array}$ & 32 & 35 & 38 & 44 & 50 & 62 & 75 & 84 & 97 & 119 & 125 & 142 & 903 \\
\hline
\end{tabular}

Graph-10: Monthly Contact Channel trend combined (Incidents \& Service Requests)

\begin{tabular}{|c|c|c|c|c|c|c|c|c|c|c|c|c|}
\hline \multicolumn{13}{|c|}{$\begin{array}{c}\text { Monthly Contact Channel trend combined (Incidents \& } \\
\text { Service Requests) }\end{array}$} \\
\hline \multirow{2}{*}{ 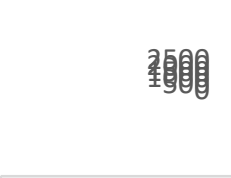 } & 2045 & 2189 & 2098 & 1897 & 2058 & 1721 & 1865 & 1576 & 1456 & 1510 & 1397 & 1340 \\
\hline & $\begin{array}{c}\text { May- } \\
19\end{array}$ & Jun-19 & Jul-19 & $\begin{array}{c}\text { Aug- } \\
19\end{array}$ & Sep-19 & Oct-19 & $\begin{array}{c}\text { Nov- } \\
19\end{array}$ & $\begin{array}{c}\text { Dec- } \\
19\end{array}$ & Jan-20 & Feb-20 & $\begin{array}{c}\text { Mar- } \\
20\end{array}$ & Apr-20 \\
\hline Tickets & 2045 & 2189 & 2098 & 1897 & 2058 & 1721 & 1865 & 1576 & 1456 & 1510 & 1397 & 1340 \\
\hline Voice & 198 & 114 & 142 & 114 & 156 & 130 & 97 & 57 & 77 & 117 & 122 & 89 \\
\hline$\longrightarrow$ Chat & 1525 & 1746 & 1644 & 1476 & 1549 & 1294 & 1365 & 1140 & 1048 & 1037 & 916 & 879 \\
\hline Email & 290 & 294 & 274 & 263 & 303 & 235 & 328 & 295 & 234 & 237 & 234 & 230 \\
\hline Self Service & 32 & 35 & 38 & 44 & 50 & 62 & 75 & 84 & 97 & 119 & 125 & 142 \\
\hline
\end{tabular}

The below table-11 and graph-11 gives an overall monthly customer contact channel ticket trend for Incidents serviced by the organization. The volume contribution for Voice is $4.5 \%$, Chat is $70 \%$, Email is $19 \%$ and Self Service is $6.5 \%$.

Table -11: Monthly Contact Channel trend (Incidents)

\begin{tabular}{|l|r|r|r|r|r|r|r|r|r|r|r|r|l|}
\hline Month & $\begin{array}{l}\text { May- } \\
19\end{array}$ & $\begin{array}{l}\text { Jun- } \\
19\end{array}$ & $\begin{array}{l}\text { Jul- } \\
19\end{array}$ & $\begin{array}{l}\text { Aug } \\
-19\end{array}$ & $\begin{array}{l}\text { Sep- } \\
19\end{array}$ & $\begin{array}{l}\text { Oct- } \\
19\end{array}$ & $\begin{array}{l}\text { Nov } \\
-19\end{array}$ & $\begin{array}{l}\text { Dec- } \\
19\end{array}$ & $\begin{array}{l}\text { Jan- } \\
20\end{array}$ & $\begin{array}{l}\text { Feb } \\
-20\end{array}$ & $\begin{array}{l}\text { Mar- } \\
20\end{array}$ & $\begin{array}{l}\text { Apr } \\
-20\end{array}$ & $\begin{array}{l}\text { Grand } \\
\text { Total }\end{array}$ \\
\hline $\begin{array}{l}\text { Inciden } \\
\mathrm{t}\end{array}$ & 1443 & $\begin{array}{r}136 \\
6\end{array}$ & $\begin{array}{r}127 \\
1\end{array}$ & $\begin{array}{r}117 \\
9\end{array}$ & $\begin{array}{r}139 \\
9\end{array}$ & $\begin{array}{r}105 \\
9\end{array}$ & 1132 & $\begin{array}{r}106 \\
7\end{array}$ & 912 & 991 & 895 & 867 & 13581 \\
\hline Voice & 114 & 48 & 56 & 35 & 75 & 52 & 28 & 23 & 17 & 76 & 77 & 30 & 631 \\
\hline Chat & 1072 & $\begin{array}{r}104 \\
7\end{array}$ & 956 & 893 & $\begin{array}{r}103 \\
4\end{array}$ & 767 & 774 & 719 & 599 & 611 & 506 & 504 & 9482 \\
\hline Email & 225 & 236 & 221 & 207 & 240 & 178 & 255 & 241 & 199 & 185 & 187 & 191 & 2565 \\
\hline $\begin{array}{l}\text { Self } \\
\text { Service }\end{array}$ & 32 & 35 & 38 & 44 & 50 & 62 & 75 & 84 & 97 & 119 & 125 & 142 & 903 \\
\hline
\end{tabular}




\section{Graph-11: Monthly Contact Channel trend (Incidents)}

\begin{tabular}{|c|c|c|c|c|c|c|c|c|c|c|c|c|}
\hline \multirow{4}{*}{$\begin{array}{r}1688 \\
138 \\
198 \\
288 \\
288\end{array}$} & \multicolumn{12}{|c|}{ Monthly Contact Channel trend (Incidents) } \\
\hline & 1443 & 1366 & 1271 & 1179 & 1399 & & & & & & & \\
\hline & \multicolumn{12}{|c|}{$\begin{array}{lllll} & 11 / 9 & 1059 & 1132 & 1067\end{array} 912$} \\
\hline & $\begin{array}{c}\text { May- } \\
19\end{array}$ & Jun-19 & Jul-19 & $\begin{array}{c}\text { Aug- } \\
19\end{array}$ & $\begin{array}{c}\text { Sep- } \\
19\end{array}$ & Oct-19 & $\begin{array}{c}\text { Nov- } \\
19\end{array}$ & $\begin{array}{c}\text { Dec- } \\
19\end{array}$ & Jan-20 & $\begin{array}{c}\text { Feb- } \\
20\end{array}$ & $\begin{array}{c}\text { Mar- } \\
20\end{array}$ & Apr-20 \\
\hline -Incident & 1443 & 1366 & 1271 & 1179 & 1399 & 1059 & 1132 & 1067 & 912 & 991 & 895 & 867 \\
\hline Voice & 114 & 48 & 56 & 35 & 75 & 52 & 28 & 23 & 17 & 76 & 77 & 30 \\
\hline Chat & 1072 & 1047 & 956 & 893 & 1034 & 767 & 774 & 719 & 599 & 611 & 506 & 504 \\
\hline Email & 225 & 236 & 221 & 207 & 240 & 178 & 255 & 241 & 199 & 185 & 187 & 191 \\
\hline Self Service & 32 & 35 & 38 & 44 & 50 & 62 & 75 & 84 & 97 & 119 & 125 & 142 \\
\hline
\end{tabular}

The below table-12 and graph-12 gives an overall monthly customer contact channel ticket trend for Service request serviced by the organization. The volume contribution for Voice is $10 \%$, Chat is $81 \%$ and Email is $9 \%$.

Table-12: Monthly Contact Channel trend (Service Requests)

\begin{tabular}{|l|l|l|l|l|l|l|l|r|r|r|r|r|l|}
\hline Month & $\begin{array}{l}\text { May- } \\
19\end{array}$ & $\begin{array}{l}\text { Jun- } \\
19\end{array}$ & $\begin{array}{l}\text { Jul- } \\
19\end{array}$ & $\begin{array}{l}\text { Aug } \\
-19\end{array}$ & $\begin{array}{l}\text { Sep- } \\
19\end{array}$ & $\begin{array}{l}\text { Oct- } \\
19\end{array}$ & $\begin{array}{l}\text { Nov- } \\
19\end{array}$ & $\begin{array}{l}\text { Dec- } \\
19\end{array}$ & $\begin{array}{l}\text { Jan- } \\
20\end{array}$ & $\begin{array}{l}\text { Feb- } \\
20\end{array}$ & $\begin{array}{l}\text { Mar- } \\
20\end{array}$ & $\begin{array}{l}\text { Apr } \\
-20\end{array}$ & $\begin{array}{l}\text { Grand } \\
\text { Total }\end{array}$ \\
\hline $\begin{array}{l}\text { Service } \\
\text { Request }\end{array}$ & 602 & 823 & 827 & 718 & 659 & 662 & 733 & 509 & 544 & 519 & 502 & 473 & 7571 \\
\hline Voice & 84 & 66 & 86 & 79 & 81 & 78 & 69 & 34 & 60 & 41 & 45 & 59 & 782 \\
\hline Chat & 453 & 699 & 688 & 583 & 515 & 527 & 591 & 421 & 449 & 426 & 410 & 375 & 6137 \\
\hline Email & 65 & 58 & 53 & 56 & 63 & 57 & 73 & 54 & 35 & 52 & 47 & 39 & 652 \\
\hline Self Service & 0 & 0 & 0 & 0 & 0 & 0 & 0 & 0 & 0 & 0 & 0 & 0 & 0 \\
\hline
\end{tabular}

\section{Graph-12: Monthly Contact Channel trend (Service Requests)}

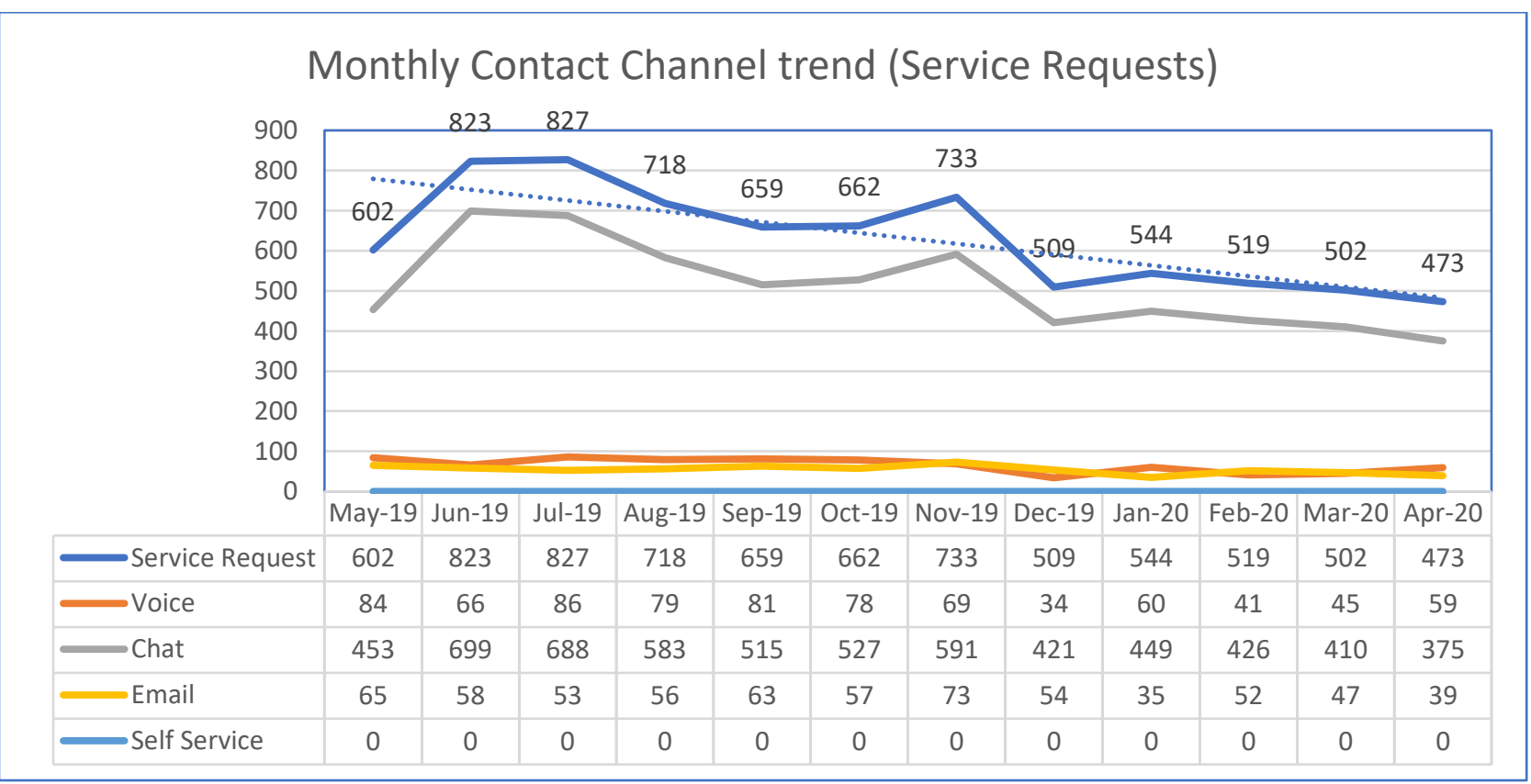




\section{FINDINGS AND RECOMMENDATIONS}

Systematic Knowledge Management implementation in the IT Service Delivery organization helps to better track the tickets raised by the customers, provides self-service opportunities for the customers and improves the support response and resolution time. Continuous Service improvement activities helps to accelerate service management and Knowledgement Management activities. The analyses reveals the ticket volume trend is getting reduced over the period of time and overall Customer satisfaction is improving month on month basis. The adoption of KM shows improvement as both Knowledge article creation and usage by the support team is evident. Self service channel is utilized for incident tickets, but the same could be leveraged for service request tickets to improve the support cycle time and further customer satisfaction.

\section{CONCLUSIONS}

The study shows Knowledge Management (KM) practice implementation and usage in IT Service Delivery Industry in India is having positive impact. Considering time, cost and effort, one of the large IT organization with 50,000 employees and having offices in multiple locations providing 10+ years of service in India was chosen for conducting the study. This study used one year primary and secondary data consisting of incidents and requests registered by the customers. The knowledge article creation and usage trend shows consistent improvement month on month basis, which is an indication of the benefits seen by the stakeholders. The customer satisfaction is consistently on the positive trend and self-service channel usage for incident reporting and resolution is encouraging. The Self-service channel could be leveraged for Service Requests as well. Overall Knowledge management contributes to the betterment of the service levels and improves the customer satisfaction. Knowledge Management creates a positive environment by having all the implicit and explicit knowledge documented, utilized, reviewed and improved as part of continuous improvement cycle.

\section{REFERENCES}

1. Santwana Chaudhuri (2011), Knowledge Management in Indian IT Industries, 3rd International Conference on Information and Financial Engineering, IACSIT Press, Singapore, IPEDR Vol 12, 2011.

2. Saraswathy and Mayakkannan, A Study on Knowledge Management about IT Sector in Chennai, International Journal in Commerce, IT \& Social Sciences, Vol 03, Issue 11, Nov 2016.

3. C.S. Ramanigopal (2012), Knowledge Management for the Oil and Gas Industry: Opportunities and Challenges, Asian Journal of Business and Economics, Vol 2, No. 2.4 Quarter IV 2012.

4. Barakath Ali Shaik Mohamed, Ramanigopal C.S and Tapesh Chandra Gupta (2020), Availability and usage of Knowledge Management (Decision support for IT and IT Enabled Services in IT Service Industry), International Journal of Advanced Science and Technology Vol 29, No. 03, 2020, 2557-2564.

5. Duraimurugan Kuppusamy and Ramanigopal C.S (2017), Knowledge Management Implementation Challenges and Opportunities in Indian Micro, Small and Medium Enterprises (MSME's), Imperial Journal of Interdisciplinary Research (IJIR), Vol 3, Issue 4, 2017.

6. Manish Kumar, Souren Paul and Suresh Tadisina (2005), Knowledge management practices in Indian software development companies: findings from an exploratory study, Asian Academy of Management Journal, Vol 10, No. 1, January 2005, 59-78.

7. Duraimurugan Kuppusamy and Ramanigopal C.S (2017), A Study on Status of Employee Awareness on Knowledge Management in Micro, Small and Medium Enterprises (MSME's) in Tamilnadu, International Research Journal of Engineering and Technology (IRJET), Vol 04 Issue 04, Apr 2017, 3196.

8. MitaliChugh, NeerajChugh, D.K. Punia and AlokAgarwal (2013), The role of information technology in knowledge management, Conference on Advances in Communication and Control Systems 2013.

9. Paween Pusaksrikit, How does Knowledge Management improve the Service Industry, jönköping international business school, jönköping university, 2006.

10. Rao R. Nemani and Steve Creason, Research Methodologies used in Knowledge Management: A Literature Review, Association for Information Systems, AIS Electronic Library (AISeL), MWAIS 2009.

11. Duraimurugan Kuppusamy, Ramanigopal C.S, Barakath Ali Shaik Mohamed (2017), Availability and Usage of Management Information System (MIS) for Knowledge Management (KM) in Micro, Small, Medium Enterprises (MSME's) in Tamilnadu, International Research Journal of Engineering and Technology (IRJET), Vol 04, Issue 04, Apr 2017. 
12. R. Udhayakumar \& P. Karthikeyan, "Adoption of Last Planner System Using Engineer's Day- Wise Card in Civil Projects for Career Advancement ", BEST: International Journal of Management, Information Technology and Engineering (BEST: IJMITE), Vol. 3, Issue 9, pp. 69-76

13. Dr. A. Vickram \& Siji Jose , "A Study on Knowledge Management Practises and its Challenges among the Blomming Companies “, International Journal of Business Management \& Research (IJBMR), Vol. 10, Issue 1, pp. 9-14

14. Swagatika Nanda, "The Role of Knowledge Management in Indian Banking Sector", IMPACT: International Journal of Research in Business Management (IMPACT: IJRBM), Vol. 4, Issue 7, pp. 37-44

15. V.Purendra Prasad \& A.Raghavendra Prasad, "Role of Knowledge Management in Indian Banking Industry (With Reference To SBI \& ICICI) “, International Journal of Educational Science and Research (IJESR), Vol. 8, Issue 1, pp. 9-18

16. S. Maria Wenisch, "Knowledge Integration Using a Cognitive Psychological Model as a Knowledge Management Strategy “, International Journal of Computer Science and Engineering (IJCSE), Vol. 6, Issue 3,pp. $45-58$

17. Benny Joseph, "An Empirical Study on Knowledge Management in Higher Educational Institutes: A Case Study of Christ Campus, Rajkot ", International Journal of Humanities and Social Sciences (IJHSS), Vol. 6, Issue 3,pp. 111 - 120 